\title{
Local Composition of Alloy Catalysts for Oxygen Reduction by STEM-EDS
}

\author{
D. Deiana ${ }^{1}$, A. Verdaguer-Casadevall ${ }^{2}$, P. Hernandez Fernandez ${ }^{2}$, F. Masini $^{2}$, C. Strebel ${ }^{2}$, D.N. \\ McCarthy $^{2}$, A. Nierhoff ${ }^{2}$, J.H. Nielsen ${ }^{2}$, I.E.L. Stephens ${ }^{2}$, I. Chorkendorff ${ }^{2}$, J.B. Wagner ${ }^{1}$ and T.W. \\ Hansen $^{1}$ \\ 1. Center for Electron Nanoscopy, Technical University of Denmark, DK-2800 Lyngby, Denmark. \\ 2. Center for Individual Nanoparticle Functionality, Technical University of Denmark, DK-2800 \\ Lyngby, Denmark.
}

Fuel cell technology is a potentially clean alternative to traditional power sources, in particular for the automotive industry [1]. The widespread usability is limited by the high cost of the Pt cathode catalyst [2]. Numerous studies therefore focus on finding cheaper alternative catalysts with higher efficiency for the oxygen reduction reaction (ORR) [3].

Bimetallic catalysts are known for their enhanced ORR activity [3,4]. $\mathrm{Pt}_{3} \mathrm{Y}$ has been identified based on density functional theory calculations as being both active and stable for ORR. Recent experimental results have shown that $\mathrm{Pt}_{\mathrm{x}} \mathrm{Y}$ in nanoparticulate form exhibit an unprecedented ORR activity of 3.05 $\mathrm{A} / \mathrm{mg}$ at $0.9 \mathrm{~V}$ with respect to a reversible hydrogen electrode [5,6]. Following the same scheme but focusing on the electrochemical oxygen reduction for the production of hydrogen peroxide, $\mathrm{Pd}-\mathrm{Hg}$ nanoparticles have been identified as a metal electrocatalyst exhibiting high mass activity $[7,8]$. In order to understand the enhanced performance of these catalysts, knowledge of how the two elements forming the bimetallic compound are distributed at the atomic level is important.

$\mathrm{Pt}_{\mathrm{x}} \mathrm{Y}$ nanoparticles have been prepared through a gas aggregation technique in a multi-chamber ultrahigh vacuum system (Omicron Multiscan Lab) while Pd-Hg nanoparticles have been synthesized by electrochemical deposition of $\mathrm{Hg}$ on $\mathrm{Pd}$ nanoparticles. The samples have been studied using high resolution Scanning Transmission Electron Microscopy (STEM) by means of a $\mathrm{C}_{\mathrm{s}}$-corrected FEI Titan 80-300. X-ray spectroscopy (X-EDS) spectrum imaging was performed on a FEI Tecnai OSIRIS in order to map the distribution of the elements in detail.

Figure 1 shows a high-resolution STEM micrograph and STEM-EDX maps of $\mathrm{Pt}_{\mathrm{x}} \mathrm{Y}$ nanoparticles that have been exposed to ORR conditions. From the micrographs, the sample presents a polycrystalline structure with a Pt rich shell and a Pt-Y core. This is ascribed to the initial dissolution of $\mathrm{Y}$ when exposed to the acidic solution of the ORR test.

Figure 2 shows analogous study for the Pd-Hg nanoparticles where a clear core-shell structure is visible from the high resolution STEM image. Since $\mathrm{Hg}$ is electrodeposited on the Pd nanoparticles, the bright contrast shell is rich of the heavy metal $\mathrm{Hg}$, while the core results composed of pure Pd as confirmed by the EDX spectrum image.

In this work, STEM-EDX mapping has been used to study in detail the elemental distribution of bimetallic nanoparticles. For the two analyzed systems a core-shell type of structure was unveiled in agreement with the theoretical predictions, making STEM-EDX spectrum imaging a powerful technique to better understand the mechanisms for the enhanced activity of these catalysts.

\section{References}

[1] M. K. Debe, Nature, 486 (2012), 43-51. 
[2] I. E. L. Stephens et al., Energy \& Environmental Science, 5 (2012), 6744.

[3] P. Strasser et al., Nature chemistry, 6 (2010), 454-60.

[4] J.K. Edward, Science 323 (2009), 1037-1041.

[5] J. Greeley et al., Nature chemistry, 7 (2010), 552-6.

[6] P. Hernandez-Fernandez et al., Submitted (2014).

[7] S. Siahrostami et al., Nature Materials 12 (2013).

[8] A. Verdaguer-Casadevall et al., Nano letters, DOI 10.1021/nl500037x (2014).

[9] The Danish National Research Foundation's Center for Individual Nanoparticle Functionality is supported by the Danish National Research Foundation. The A.P. Moller and Chastine Mc-Kinney Møller Foundation is gratefully acknowledged for its contribution towards the establishment of the Centre for Electron Nanoscopy in the Technical University of Denmark. The Interdisciplinary Centre for Electron Microscopy (CIME) at EPFL is gratefully acknowledged for the use of the FEI Tecnai Osiris TEM.
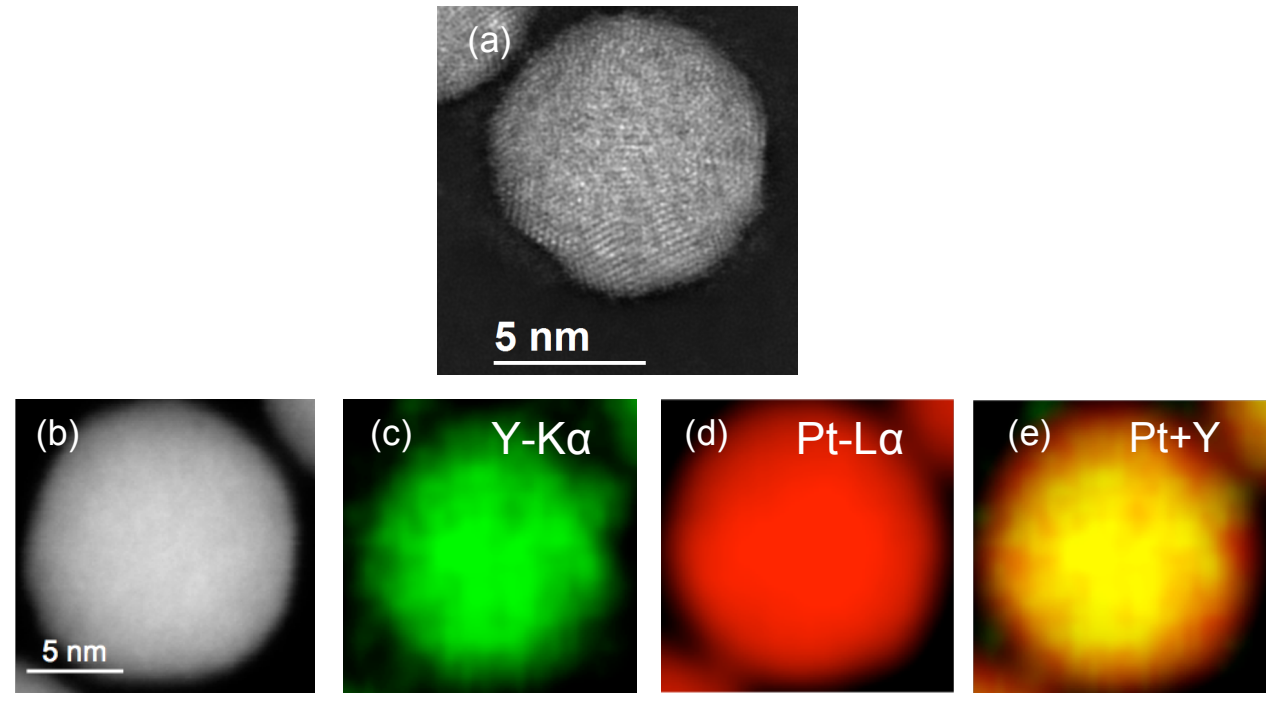

Figure 1. a) High resolution STEM micrograph of a $\mathrm{Pt}_{\mathrm{x}} \mathrm{Y}$ nanoparticle. b) STEM micrograph of a nanoparticle and b-d) corresponding Y, Pt and combined X-Ray elemental maps.
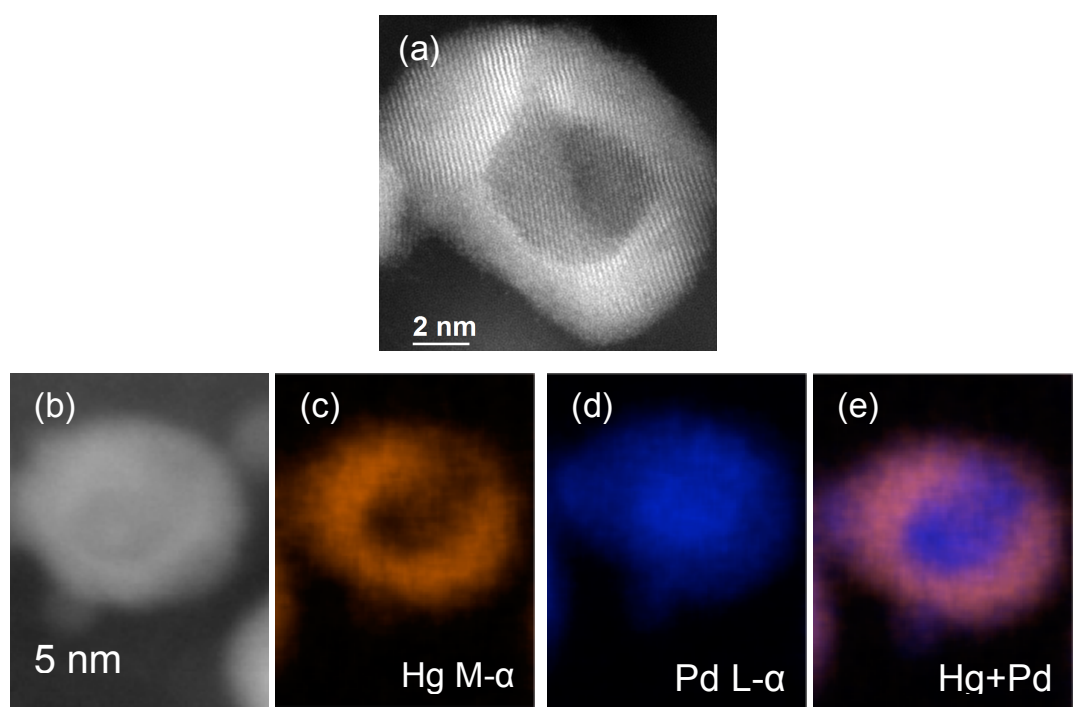

Figure 2. a) High resolution STEM micrograph of a Pd-Hg nanoparticle. b) STEM micrograph of a nanoparticle and b-d) corresponding $\mathrm{Hg}$, Pd and combined X-Ray elemental maps. 